\title{
Effective mixing in a microfluidic chip using magnetic particles $\dagger$
}

\author{
Seung Hwan Lee, $\ddagger^{a}$ Danny van Noort, $\sharp^{b c}$ Ji Youn Lee, ${ }^{a d}$ Byoung-Tak Zhang ${ }^{b}$ and Tai Hyun Park ${ }^{* a}$ \\ Received 18th August 2008, Accepted 17th October 2008 \\ First published as an Advance Article on the web 18th November 2008 \\ DOI: 10.1039/b814371d
}

We present a novel active mixing method in a microfluidic chip, where the controlled stirring of magnetic particles is used to achieve an effective mixing of fluids. To perform mixing, the ferromagnetic particles were embedded and manipulated under the influence of a rotating magnetic field. By aligning the magnetic beads along the magnetic field lines, rod-like structures are formed, functioning as small stir bars. Under higher flow conditions the particles did not form the typical rod structure but rather formed aggregates, which were even more beneficial for mixing. Our system reached a $96 \%$ mixing efficiency in a relatively short distance $(800 \mu \mathrm{m})$ at a flow rate of $1.2-4.8 \mathrm{~mm} / \mathrm{s}$. These results demonstrate that our mixing method is useful for microfluidic devices with low aspect ratios and molecules with large molecular weights.

\section{Introduction}

Rapid mixing in microfluidic channels is difficult due to laminar flows. Mixing of flows is dominated by diffusion, meaning a sufficient channel length is needed for complete mixing. ${ }^{1-5}$ Mixing problems can broadly be divided into two classes: passive and active mixing. Previous studies have suggested that active mixing is more effective than passive mixing. ${ }^{6}$ Recently, both passive and active mixing devices utilizing beads in microfluidic channels have been introduced. ${ }^{7,8}$

In this article, we demonstrate active mixing in a microfluidic device based on the controlled stirring of magnetic particles. Through the use of pneumatic valves, the particles were retained to a designated region in the device. Since magnets and an electrical stirrer were used to generate a rotating magnetic field, the chip size and aspect ratio could be down-scaled. Magnetic particles in the microfluidic chamber rotated by following the changing magnetic field pattern. The controlled magnetic stirring of the particles showed a more efficient mixing than passive mixing would have.

\section{Experimental}

\section{Chip design and microfabrication}

The design of the microfluidic device for chaotic mixing is outlined in Fig. 1. The microfluidic device consists of two layers. The

${ }^{a}$ School of Chemical and Biological Engineering, Institute of Bioengineering, Seoul National University, Seoul, 151-744, Korea. E-mail: thpark@snu.ac.kr; Fax: +82-2-875-9348; Tel: +82-2-880-8020

${ }^{b}$ School of Computer Science and Engineering, Seoul National University, Seoul, 151-744, Korea

'Institute of Bioengineering and Nanotechnology, 31 Biopolis Way, The Nanos \#04-01, Singapore 138669

${ }^{d}$ Department of Biomedical Engineering, and Department of Medicine, Transplant Research Institute, University of California, Davis, California 95616, USA

$\dagger$ Electronic supplementary information (ESI) available: Experimental setup (Fig. S1); image of the chamber containing magnetic particles (Fig. S2). See DOI: 10.1039/b814371d

\$ The authors contributed equally to this work. bottom layer is a Y-shaped channel containing the mixing chamber and barriers. The channel is $100 \mu \mathrm{m}$ wide and $10 \mu \mathrm{m}$ deep. The chamber is $35 \mu \mathrm{m}$ deep and the space above the bead barriers is $3 \mu \mathrm{m}$. The barriers are located in the three channels around the chamber to keep the beads in place, except for the particle solution inlet channel. The top layer has pneumatic channels (100 $\mu \mathrm{m}$ wide, $12.5 \mu \mathrm{m}$ deep) and valves $(300 \mu \mathrm{m} \times 550$ $\mu \mathrm{m} \times 12.5 \mu \mathrm{m})$ to control the fluid. The chip was fabricated by using standard multi-layer soft-lithography., ${ }^{9,10}$

\section{Device operation}

The device operation was described elsewhere. ${ }^{11}$ The rotor from a commercial stirrer was used to rotate the magnetic particles. The outer cover of the stirrer was removed and two NeodymiumIron-Boron magnets (NdFeB, Magtopia, Gumi, Korea) were placed onto the rotor. The whole setup was then placed under a fluorescent stereo microscope (SV-6, Zeiss Optics, Jena, Germany) equipped with a Peltier cooled CCD camera to monitor the changes in the flow patterns (see Fig. S1 in the ESI for details $\dagger$ ).

\section{Mixing experiment}

To monitor mixing, a fluorescent solution was obtained by dissolving fluorescein isothiocyanate dextran, $30 \mu \mathrm{M}$ (FITCdextran, MW 70000, Sigma Chemical Company, St. Louis, MO, USA), directly in Tris-HCl buffer ( $\mathrm{pH}=7.6)$. First, the magnetic particle control valve (valve No. 2 in Fig. 1B) was closed and a non-fluorescent buffer was pumped through the fluid inlet channel to remove all bubbles in the device. Then, the flow was blocked by closing the fluid control valve (valve No. 1 in Fig. 1B). After opening the magnetic particle control valve, ferromagnetic particles (4 $\mu \mathrm{m}$ diameter, Spherotech, Inc., Lake Forest, IL, USA) were introduced into the chamber by pumping 3-5 $\mu$ l of magnetic particle suspension $(1 \% \mathrm{w} / \mathrm{v})$ through the magnetic particle channel using a syringe. Then, the magnetic particle control valve was closed, and the fluid control valve was opened. The resulting fluorescent distribution profile over the channel's width was then monitored with rotating or non-rotating 
(A)

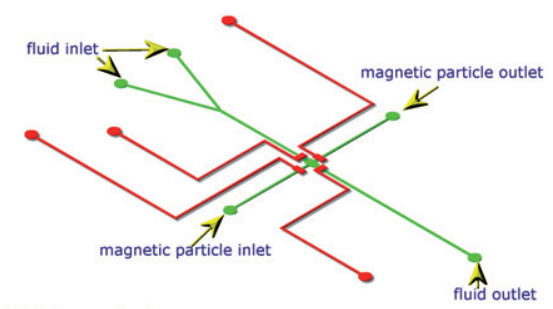

(B)

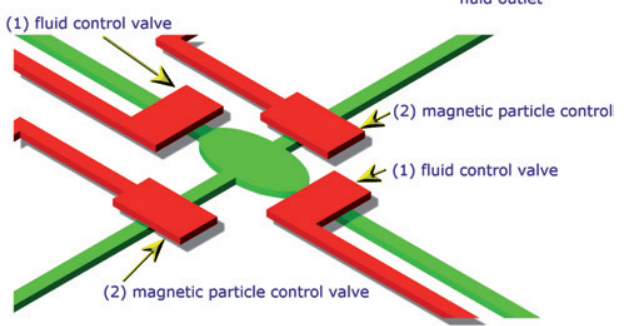

Fig. 1 (A) Schematic of the mixing chip, The green lines represent the channels in the bottom layer, and the red lines represent the pressure lines for the control of the pneumatic valves in the top layer. (B) Close up of the mixing chamber; (1) the valve for the fluid control and (2) the valve for the magnetic particle control.

magnetic particles. To evaluate the mixing performance, the mixing efficiency was calculated using the mixing efficiency equation. ${ }^{12}$

\section{Results and discussion}

Ferromagnetic particles retain their magnetism and stay in aggregated form even in the absence of the magnetic field. Therefore, the ferromagnetic particles are more advantageous than superparamagnetic particles for active mixing. Because the space above the bead barrier is $3 \mu \mathrm{m}$, smaller particles pass through this space. When larger particles (i.e. $7 \mu \mathrm{m}$ ) were used, they were not smoothly rotated in the mixing chamber and some were stuck in the particle inlet channel. For these reasons the proper particle size was $4 \mu \mathrm{m}$ for the dimensions of this device. The amount of particles in a mixing chamber is also critical for effective mixing. Injection of $3-5 \mu 1$ of $1 \% \mathrm{w} / \mathrm{v}$ particle solution into the chamber supplied an appropriate amount of particles.
For a given flow rate, the mixing phenomenon is different at different aspect ratio of the channel. When two fluids flow are adjacent to one another in the microchannel, diffusion occurs at the interface of the two fluids. High aspect ratio means large contact area between the fluids, whereas low aspect ratio means a smaller contact area between fluids. In our case, the aspect ratio is 0.0875 in the chamber, and 0.1 in the channel, which is a hard mixing condition.

The NdFeB magnets were placed on the rotor of the stirrer to generate a large enough magnetic field and to rotate particles against the viscous drag force of flow. A field strength of $0.54 \mathrm{~T}$ was obtained above the magnets. The magnetic particles were exposed to the external magnetic field, acquiring a magnetic dipole moment and aligning along the magnetic field lines (see Fig. S2-A in the ESI $\dagger$ ). When the NdFeB magnets rotated, the rod structure of the coalesced magnetic particles correspondingly rotated due to a changing magnetic field. The observed dynamics of magnetic chains in a rotating magnetic field has been reported in previous studies. ${ }^{8,13,14}$ Images were taken at a 0.16 second time intervals to trace the rotational motion of the magnetic particles in the absence of a flow (Fig. 2A, B, C). These images clearly show the rod structure of magnetic particles and their rotating. Fig. 2A, 2B show the micro particles rotating at 120 and 200 RPM, respectively. As the rotation speed increased, the length of the rod structures became shorter. When rotating at 500 RPM, the particles did not form the typical rod structure in the chamber but rather formed aggregates (Fig. 2C). Their irregular shape, relatively larger size, and their irregular motion were beneficial for effective mixing. To study the motion of particles under flow, the flow control valve was opened and the movement of particles was monitored (see Fig. S2-B in the ESI $\dagger$ ). Some of the particles could not withstand the viscous drag of the flow and were pushed against the barrier forming a packed mixer. The remaining aggregates in the chamber displayed controlled magnetic stirring. This means that both passive and active mixing are incorporated in this system. These pushed out particles did not block the barrier opening in this flow rate range. However, at a flow rate higher than $4.8 \mathrm{~mm} / \mathrm{s}$, the particles clumped together and did not rotate.

To characterize mixing of two fluids by magnetic particles in this device, fluorescent and non-fluorescent solution were
(A)

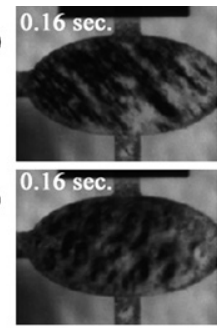

(C)

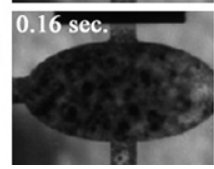

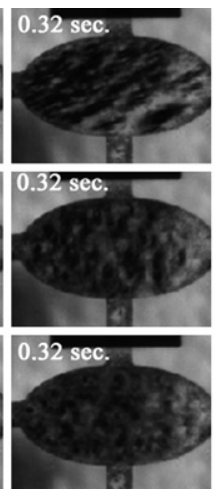
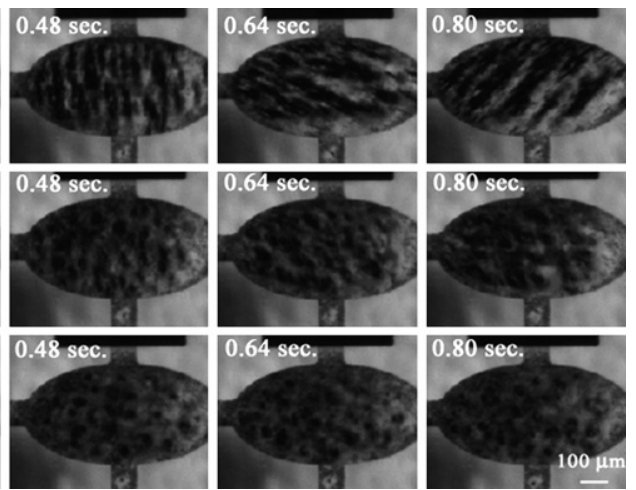

Fig. 2 Images of the rotating magnetic particles taken at $0.16 \mathrm{sec}$. intervals for different stirrer RPM. (A) Columnar structure of magnetic particles rotating counter-clockwise at 120 RPM of stirrer. (B) Shortened columnar structure of magnetic particles at 200 RPM (compare with Fig. 2. (A)). At $0.16,0.48,0.64$ sec., the direction of particles is different. (C) Irregular shape of aggregated magnetic particles at 500 RPM. 
(A)

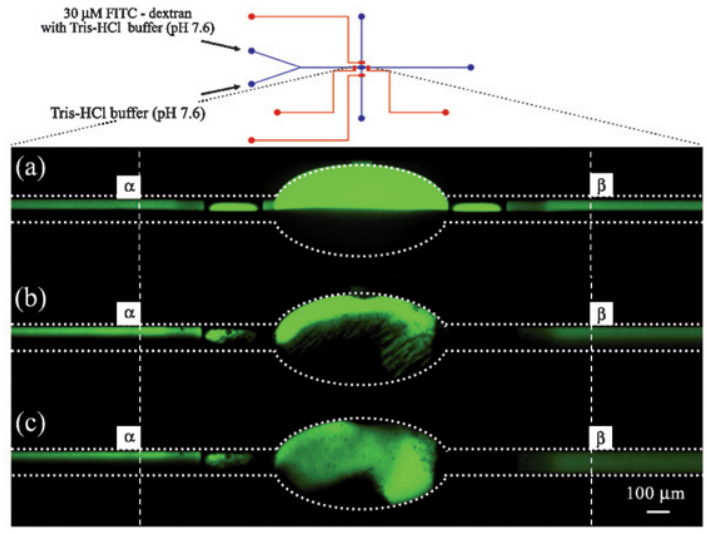

(B)

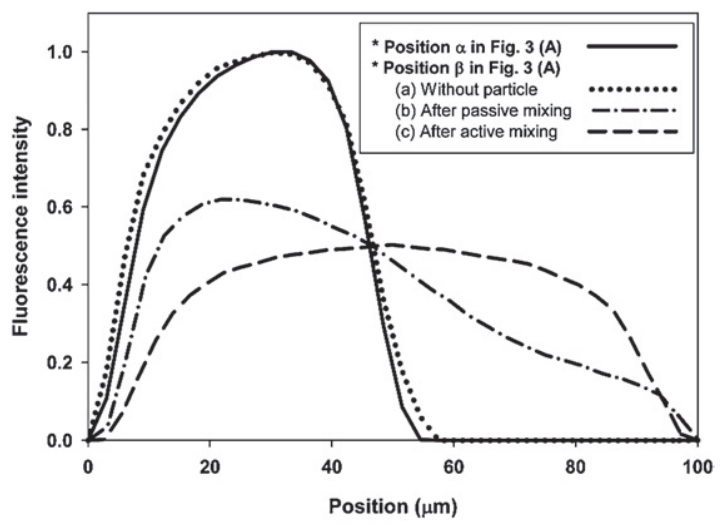

Fig. 3 (A) Schematic representation of the mixing chip and fluorescence micrograph (a) Fluorescence micrograph of the mixing chamber without magnetic particles; The location of the fluorescence intensity scan lines is indicated by the dashed line (See the dotted line in Fig. 3B, without particles). $\alpha$ is before the mixing chamber and laminar flow is observed. $\beta$ is after the mixing chamber and laminar flow does not disrupt. (b) Fluorescence micrograph of the mixing chamber with non rotating magnetic particles; The location of the fluorescence intensity scan lines is indicated by the dashed line (See the dash - dotted line in Fig. 3B, passive mixing). $\alpha$ is before the mixing chamber and laminar flow is observed. $\beta$ is after the mixing chamber and mixing is not complete. (c) Fluorescence micrograph of the mixing chamber containing rotating magnetic particles; The location of the fluorescence intensity scan lines is indicated by the dashed line (See the dashed line in Fig. 3B, active mixing). $\alpha$ is before the mixing chamber and laminar flow is observed. $\beta$ is after the mixing chamber and efficient mixing is observed. (B) Fluorescent intensity profile of a FITC-dextran dye solution across the transverse direction of the channel at a flow rate of $1.2-4.8 \mathrm{~mm} / \mathrm{sec}$

pumped through the respective inlets of the Y-shaped channel at a flow rate of $1.2-4.8 \mathrm{~mm} / \mathrm{s}$. As the flows are laminar, mixing is only possible by diffusion. In our system, the diffusion time was calculated to be $71.43 \mathrm{sec}$ by Einstein's formula of Brownian motion. ${ }^{6}$ The required mixing distance needed is $86 \mathrm{~mm}$ at the minimum flow rate of $1.2 \mathrm{~mm} / \mathrm{s}$ up to $343 \mathrm{~mm}$ at $4.8 \mathrm{~mm} / \mathrm{s}$. In the absence of beads, the laminar flow pattern was not mixed by the barrier or chamber structure (Fig. 3A-a- $\beta$ ). With non rotating magnetic particles in the chamber, the laminar flow pattern was disturbed due to the high random porosity of packed and suspended particles (Fig. 3A-b- $\beta$ ). Under these conditions two solutions were not completely mixed. The passive mixing is effective in promoting longitudinal rather than lateral mixing. ${ }^{6}$ Fig. 3A-c shows the fluorescent intensity of the flow when magnetic particles are subjected to controlled stirring. The FITCdextran solution and buffer solution were completely mixed (Fig. 3A-c- $\beta$ ). A clear difference in mixing performance was obtained between the two cases (Fig. 3A-b, A-c). This means that rotation and irregular motion of the magnetic particles in low aspect ratio channel can solve the problem of the poor mixing efficiency seen in the passive mixing of particles. To quantitatively analyze the mixing efficiency, the fluorescent intensity distribution across the channel in transverse direction was measured (Fig. 3B). The mixing efficiency was almost zero before and after entering the chamber containing no magnetic particles. When the particles were not rotating in the chamber, a mixing efficiency of $66 \%$ was obtained. By rotating magnetic particles the mixing efficiency was increased to $96 \%$ and this efficiency was generated over a relative short distance $(800 \mu \mathrm{m})$. This occurs because controlled magnetic stirring promotes chaotic fluid streams with fluctuating longitudinal and lateral mixing.

\section{Conclusions}

We presented the design and fabrication of a novel mixing device which uses the controlled stirring of ferromagnetic particles to promote active mixing. Because of the low Reynolds number in the microchannel, active mixing is required to improve mixing performance in a relative short channel length. The dynamic and irregular motion of the particles was observed by rotating $\mathrm{NdFeB}$ magnets. Efficient mixing results were obtained at relative fast flow rates (up to $4.8 \mathrm{~mm} / \mathrm{s}$ ). These results indicate that lateral directional mixing is enhanced by the controlled stirring of magnetic particles. Also, this concept overcomes the limits of passive and active mixing. Finally, our device displayed a high efficiency of mixing for larger molecules (similar in size to those present in biological experiments) and in a low aspect ratio system. Since the ferromagnetic particles were coated with a carboxyl group, subsequent surface modifications or attachment of biological materials would be possible, thus increasing the applicability of this microfluidic chip. As these particle are biocompatible and do not contaminate the solution, this mixing method can be applied to various biological fields including glucose sensor, ${ }^{7}$ PCR (polymerase chain reaction) and continuous flow fast reaction kinetic study.

\section{Acknowledgements}

This work was supported by a grant from the Innovative Research Institute for Cell Therapy (No. A06-2260-B8150506N1-15010A) and by the KOSEF through the Nano-Bioelectronics and Systems Research Center, Seoul National University. This work was also supported in part by MICE through the Molecular Evolutionary Computing (MEC) Project.

\section{References}

1 S. C. Jacobson, T. E. McKnight and J. M. Ramsey, Anal. Chem., 1999, 71, 4455-4459.

2 A. E. Kamholz, B. H. Weigl, B. A. Finlayson and P. Yager, Anal. Chem., 1999, 71, 5340-5347.

3 S. K. W. Dertinger, D. T. Chiu, N. L. Jeon and G. M. Whitesides, Anal. Chem., 2001, 73, 1240-1246. 
4 R. F. Ismagilov, D. Rosmarin, P. J. A. Kenis, D. T. Chiu, W. Zhang, H. A. Stone and G. M. Whitesides, Anal. Chem., 2001, 73, 4682-4687.

5 P. J. A. Kenis, R. F. Ismagilov, S. Takayama, G. M. Whitesides, S. Li and H. S. White, Acc. Chem. Res., 2000, 33, 841-847.

6 B. He, B. J. Burke, X. Zhang, R. Zhang and F. E. Regnier, Anal. Chem., 2001, 73, 1942-1947.

7 G. H. Seong and R. M. Crooks, J. Am. Chem. Soc., 2002, 124, 13360 13361.

8 A. Rida and M. A. M. Gijs, Anal. Chem., 2004, 76, 6239-6246.

9 M. A. Unger, H. P. Chou, T. Thorsen, A. Scherer and S. R. Quake, Science, 2000, 288, 113-116.
10 J. K. Chang, Y. S. Heo, H. Bang, K. Cho, S. Chung, C. Chung and D. C. Han, Biotechnol. Bioprocess Eng., 2003, 8, 233-239.

11 S. -W. Nam, D. V. Noort, Y. Yang and S. Park, Lab Chip, 2007, 7, 638-640.

12 N. L. Jeon, S. K. W. Dertinger, D. T. Chiu, I. S. Choi, A. D. Stroock and G. M. Whitesides, Langmuir, 2000, 16, 8311-8316.

13 S. Melle, O. G. Calderon, G. G. Fuller and M. A. Rubio, J. Colloid Interface Sci., 2002, 247, 200-209.

14 A. K. Vuppu, A. A. Garcia and M. A. Hayes, Langmuir, 2003, 19, $8646-8653$. 